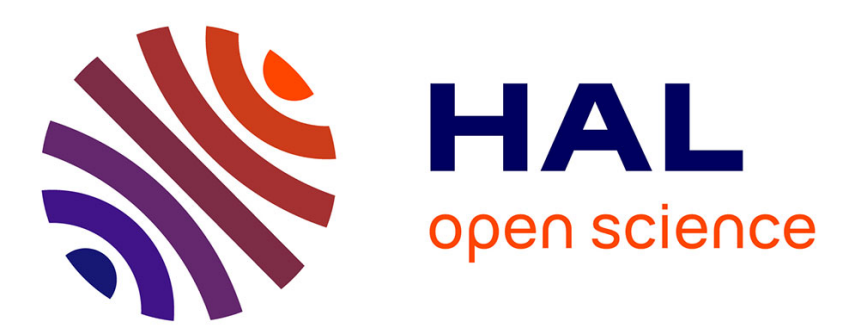

\title{
HgTe Nanocrystal Inks for Extended Short-Wave Infrared Detection
}

Bertille Martinez, Julien Ramade, Clément Livache, Nicolas Goubet, Audrey

Chu, Charlie Gréboval, Junling Qu, William L Watkins, Loic Becerra, Erwan

Dandeu, et al.

\section{To cite this version:}

Bertille Martinez, Julien Ramade, Clément Livache, Nicolas Goubet, Audrey Chu, et al.. HgTe Nanocrystal Inks for Extended Short-Wave Infrared Detection. Advanced Optical Materials, 2019, pp.1900348. 10.1002/adom.201900348 . hal-02134751

\section{HAL Id: hal-02134751 \\ https://hal.science/hal-02134751}

Submitted on 10 Jul 2020

HAL is a multi-disciplinary open access archive for the deposit and dissemination of scientific research documents, whether they are published or not. The documents may come from teaching and research institutions in France or abroad, or from public or private research centers.
L'archive ouverte pluridisciplinaire HAL, est destinée au dépôt et à la diffusion de documents scientifiques de niveau recherche, publiés ou non, émanant des établissements d'enseignement et de recherche français ou étrangers, des laboratoires publics ou privés. 


\title{
HgTe Nanocrystal Inks for Extended Short Wave Infrared Detection
}

Bertille Martinez ${ }^{1,2}$, Julien Ramade ${ }^{1}$, Clément Livache ${ }^{1,2}$, Nicolas Goubet ${ }^{1,2}$, Audrey $\mathrm{Chu}^{1}$, Charlie Gréboval ${ }^{1}$, Junling Qu ${ }^{1}$, William L. Watkins ${ }^{1}$, Loïc Becerra ${ }^{1}$, Erwan

Dandeu $^{1}$, Jean Louis Fave ${ }^{1}$, Christophe Méthivier ${ }^{3}$, Emmanuelle Lacaze ${ }^{1}$, Emmanuel Lhuillier $^{1^{*}}$

1Sorbonne Université, CNRS, Institut des NanoSciences de Paris, INSP, F-75005

Paris, France

'Laboratoire de Physique et d'Étude des Matériaux, ESPCI-ParisTech, PSL Research University, Sorbonne Université UPMC Univ Paris 06, CNRS, 10 rue Vauquelin 75005 Paris, France

${ }^{3}$ Sorbonne Université, CNRS, Laboratoire de Réactivité de Surface, LRS, F-75005 Paris, France

\begin{abstract}
Short wave infrared (IR) detection is currently driven by InGaAs technology which has limited the perspective of cost effectiveness and consequently slowed the development of IR sensors. Since organic electronics are ineffective in this wavelength range, an alternative to conductive polymers is the use of colloidal quantum dots (CQDs) which exhibit strongly tunable IR absorption. In this paper, we focus on the extended short-wave IR ( $2.5 \mu \mathrm{m}$ cut-off) to expand the capabilities of InGaAs, while using HgTe nanocrystals as the active material. Previous devices based on this material suffered from a low responsivity due to weak absorption (few percents). We present the integration of HgTe nanocrystals in ink form to build thick (up to $600 \mathrm{~nm}$ ), strongly absorbing nanoparticle films. This ink is integrated into a diode, allowing one to boost the responsivity by two orders of magnitude and the detectivity by one order of magnitude compared to previous HgTe devices at the same wavelength. Detectivity reaches $3 \times 10^{9}$ Jones, while the time response is found to be $370 \mathrm{~ns}$ for room temperature and $0 \mathrm{~V}$ bias operation. Finally, the material is integrated into a focal plane array which is used to determine laser beam profile.
\end{abstract}

Keywords: HgTe, nanocrystals, short wave infrared, photodiode, focal plane array.

To whom correspondence should be sent: el@insp.upmc.fr 
TOC Graphic

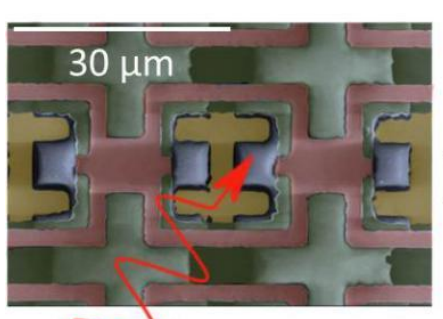

$\int \mathrm{hv} 1,55 \mu \mathrm{m}$

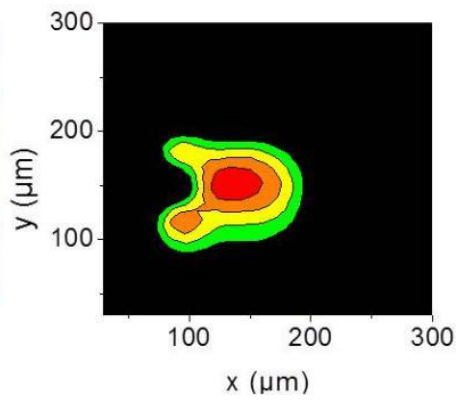

TOC text:

We use ink made from HgTe nanocrystals to fabricate strongly absorbing film in the extended short wave infrared. This material is then integrated into photodiode and focal plane array 


\section{INTRODUCTION}

The interest for nanocrystals ${ }^{[1-3]}$ has been mostly driven by their luminescent properties in applications such as displays, ${ }^{[4]}$ single photon sources ${ }^{[5]}$ or fluorescent imaging, ${ }^{[6]}$ though their absorption features appear equally promising. In particular, nanocrystal absorption is a robust property (i.e. absorption barely photobleaches and is not strongly affected by a change in the dielectric environment or surface chemistry) which can be broadly tuned from UV to THz ranges. ${ }^{[7-11]}$ This robustness combined with the possibility to design narrow energy transition absorption which, unlike organic electronics, enables nanocrystals to be used for infrared sensing.

Short wave infrared (SWIR: 1-3 $\mu \mathrm{m}$ ) is a part of the electromagnetic spectrum invisible to the naked eye finding applications in night vision, LIDAR imaging or active imaging. Infrared sensing in the SWIR is currently led by InGaAs semiconductors. The high performances achieved by this material are currently strongly inhibited by its cost. Indeed, road maps for this technology only plan marginal cost reduction. InGaAs also suffers from a lack of spectral tunability, especially when wavelengths longer than $1.7 \mu \mathrm{m}$ are required. Significant efforts have been dedicated in the design of alternative infrared sensors based on colloidal nanocrystals. PbS has demonstrated significant performances in the near IR and up to telecom wavelengths. ${ }^{[12,13]}$ Nevertheless, above $1.5 \mu \mathrm{m}$, quite large nanoparticles have to be grown exhibiting lower colloidal stability, ${ }^{[14]}$ which is highly detrimental for the design of pinhole-free vertical geometry devices. An alternative is to use narrower band gap semiconductors and semimetals. ${ }^{[15,16]}$ Under colloidal form, $\mathrm{HgTe}$ is certainly the most mature material combining infrared absorption and photoconduction. ${ }^{[17]}$ On the other hand, efforts dedicated to the integration of HgTe nanocrystals into photodiodes ${ }^{[18,19]}$ are much fewer than those reported for $\mathrm{PbS}$. As a result, most devices based on HgTe nanocrystals with long wavelength absorption are based on planar

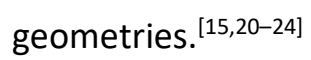

Recently, mid-wave infrared photodiodes ${ }^{[25]}$, with high photoresponse ${ }^{[26]}$ (up to $0.8 \mathrm{~A} / \mathrm{W}$ ), have been proposed by Guyot-Sionnest's group. Part of this success is based on the use of plasmon resonance to enhance the absorption of thin nanocrystal films with thicknesses below the absorption depth. ${ }^{[20,21,26,27]}$ Yet, their $5 \mu \mathrm{m}$ cut-off wavelength means that these devices still need to be cooled down to cryogenic temperatures to achieve a reasonable signal/noise ratio. In the SWIR and extended SWIR (up to $2.5 \mu \mathrm{m}$ ), targeted performances need to be achieved without cooling or with a Peltier system at most. Thus, new designs must be proposed. In this wavelength range, Jagtap et $a^{[28,29]}$ have proposed the use of a basic diode structure inspired by solar cells : a solid-state ligand exchanged $\mathrm{HgTe}$ layer is coupled with a $\mathrm{TiO}_{2}$ electron transport layer which is used to induce the rectifying behavior. Later, it was proposed to add a unipolar barrier which had the purpose to selectively decrease the electron dark current. Room temperature detectivity up to $3 \times 10^{8}$ Jones has been demonstrated. ${ }^{[23]}$ In this device, the performance remains clearly limited by the low responsivity $(100 \mu \mathrm{A} / \mathrm{W}$ at $0 \mathrm{~V})$ rather than by the signal/noise ratio. Indeed, the $\mathrm{TiO}_{2}$ layer, with its $3.2 \mathrm{eV}$ band gap used for the diode, presents a poor band alignment with HgTe nanocrystals. As a result, not only is the dark hole current filtered but so is a significant part of the photocurrent. Furthermore, only thin films ( $<200 \mathrm{~nm}$ ) of HgTe have been used as absorbing layers leading to a low absorption. The absorption coefficient ${ }^{[30]} \alpha$ of HgTe nanocrystal with a cut-off at $2.5 \mu \mathrm{m}$ has been estimated to be $3 \pm 1 \times 10^{3} \mathrm{~cm}^{-1}$, thus we can estimate the absorption of a $200 \mathrm{~nm}$ thin film to be 1$10^{-\alpha L} \approx 12 \%$. Indeed, solid state ligand exchange, which is generally used for HgTe nanocrystals devices, makes it challenging to fabricate thick, smooth and strongly electronically coupled films. In 
the case of PbS, the recent trend is to switch from the deposition of nanocrystals in an apolar solvent to nanocrystal inks ${ }^{[31-33]}$ where ligand exchange is undertaken through a phase transfer. ${ }^{[34]}$ This effort is still quite marginally used for HgTe. Here, we explore the potential of a HgTe nanocrystal-based ink for the design of extended SWIR sensors. The capability of this ink is then tested for the design of a photodiode and a multipixel device. We particularly demonstrate the capability of this focal plane array to determine the infrared laser beam shape.

\section{DISCUSSION}

At first, $\mathrm{HgTe}$ nanocrystals were synthesized with an excitonic feature at $4000 \mathrm{~cm}^{-1}$ ( $2.5 \mu \mathrm{m}$ or $0.5 \mathrm{eV}$ ) using the Keuleyan's procedure. ${ }^{[35]}$ The nanoparticles, later named HgTe $4 \mathrm{k}$, undertake a tetrapodic shape, as shown in the transmission electron microscopy (TEM) image in Figure 1a. Secondly, an ink was prepared from this solution. Since mercury has a strong affinity with thiols, a short thiol (ethanedithiol or mercapto alcohol) was used as a transfer agent to disperse the nanocrystals in the polar phase solvent dimethyl formamide (DMF) from the initial toluene solvent. The addition of $\mathrm{HgCl}_{2}$ was found to further enhance electronic passivation and allowed good conductivity. ${ }^{[25,36-38]}$ We observed that the nanoparticle spectrum was barely affected by this ligand exchange procedure, see Figure $1 \mathrm{~b}$. This contrasts with the solid state ligand exchange (SSLE) based on ethanedithiol (EDT) which led to a redshift and broadening of the band edge transition. ${ }^{[39]}$ Spin coating of this solution at low speeds (<1000 rpm) yielded thick (up to $600 \mathrm{~nm}$ ) and smooth films of HgTe, with a roughness of the order of the nanocrystal size, see Figure 1c. A vacuum drying step was then required to remove all the left over DMF.

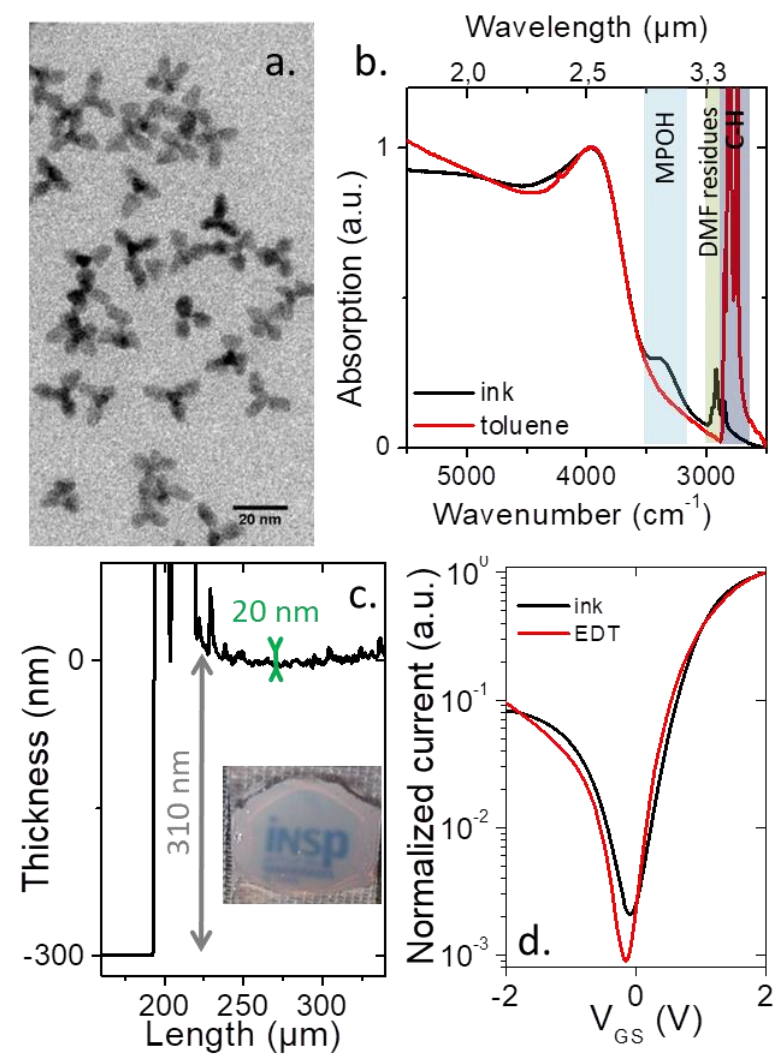


Figure 1 : a) TEM image of HgTe $4 \mathrm{k}$ used in this study. b) Infrared spectra of HgTe 4k as-synthetized (DDT ligands, toluene, in red) and ligand-exchanged (ink, DMF, in black). c) Profilometry of a HgTe ink film, insert: macroscopic image of HgTe ink film. The film the quality of the film is highlighted by its reflectivity. d) Transfer curves measured for ink device (in black) and EDT on film ligand exchange device (in red). The two curves were normalized by their value at $+2 \mathrm{~V}$. The current leakage was at least one order of magnitude below the channel current (see Figure S4b and S4c).

The transport properties of the layer, probed in an electrolyte gated field effect transistor configuration, ${ }^{[40]}$ shows that the ambipolar character of the film is mostly unaffected, see Figure $1 \mathrm{~d}$ and Figure S4. However the mobility of the film is significantly improved, see Figure S5. The determination of the mobility was done using conventional back gating through a $\mathrm{SiO}_{2}$ layer. We determined the electron mobility to be $3 \times 10^{-2} \mathrm{~cm}^{2} \mathrm{~V}^{-1} \mathrm{~s}^{-1}$, while the mobility of HgTe nanocrystals capped with EDT was previously determined ${ }^{[39]}$ to be $10^{-4} \mathrm{~cm}^{2} \mathrm{~V}^{-1} \mathrm{~s}^{-1}$. This is indeed promising to achieve longer diffusion lengths and collect photocarriers over a thicker layer.

In addition, the ink exhibits an enhanced stability compared to SSLE prepared films, see Figure 2a. Indeed, the resistance of a film stored in ambient conditions over a week only dropped by a factor two, while a factor 1000 drop was observed for a SSLE prepared film. With the SSLE process, coating the device with water and oxygen repealing layers was mandatory to achieve stable performances. ${ }^{[28]}$ Understanding the increase in dark current, gives insight on how the optical feature diminishes over time. The film was annealed at $120^{\circ} \mathrm{C}$, to mimic clean room processing. This temperature is similar to what it is expected to be exposed during the lithography process or atomic layer deposition process. With conventional SSLE with EDT, we observed a severe redshift of the exciton, see Figure 2c, while the energy of the band edge remains almost unaffected for the ink, see Figure $2 b$. Indeed, upon aging and annealing, HgTe nanocrystals prepared by SSLE sinter which reduces the confinement and the optical band gap. As the dark current is exponentially dependent on the band gap, the reduced band gap leads to an increase of the dark current.

Films prepared by SSLE EDT and inks were also analyzed before and after aging using photoemission spectroscopy. As the ratio $\mathrm{Te} / \mathrm{Hg}$ is constant in the case of ink based films, it increases by $63 \%$ for SSLE EDT films. On these SSLE films, we see a new contribution of Te $3 \mathrm{~d}$ core levels at $576.1 \mathrm{eV}$ appearing after 72 hours aging, see Figure 2e. This contribution can be attributed to the formation of $\mathrm{TeO}_{2 .}{ }^{[41]}$ Mercury core levels were also investigated, but neither aging nor preparation methods (SSLE or ink) had a significant influence, see Figure $2 \mathrm{~d}$.

In the case of SSLE films, at low binding energies, the valence band shifts towards the Fermi level, see Figure S3b and c. This shift leads to a p-type doping consistent with the increase of Te/Hg ratio. On films prepared by ink, the energy difference between the valence band and Fermi level stays constant after 72 hours of aging and no oxidation of tellurium was observed. The p-doping of the nanocrystals also participates in the increase of dark current in the case of SSLE preparation. 

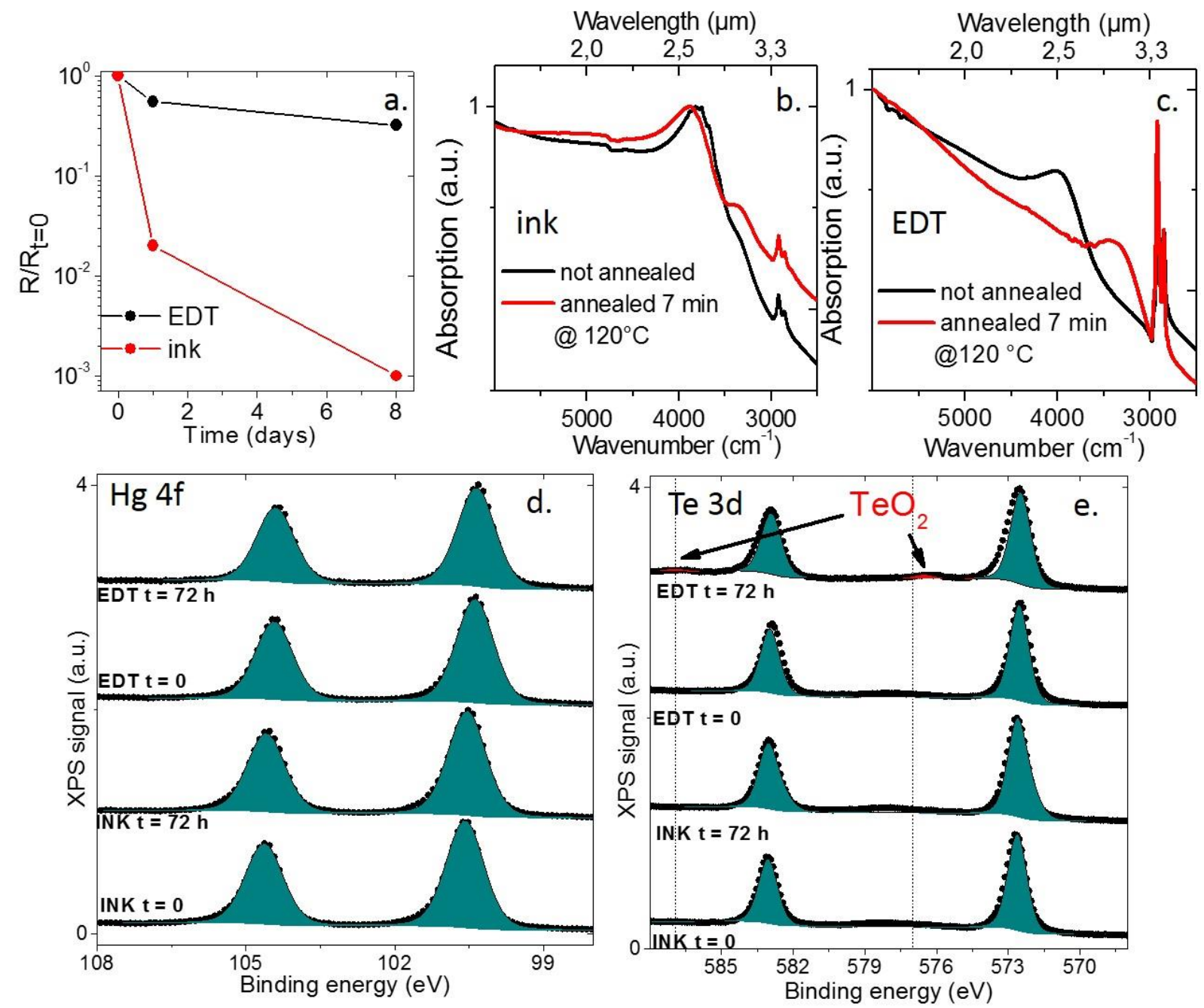

Figure 2: a) Evolution of resistance under $1 \mathrm{~V}$ bias for two photoconductive devices: ink device (in black) and EDT on film ligand exchange (in red). b) Absorption spectrum of a HgTe nanocrystal film obtained from an ink before and after annealing at $120^{\circ} \mathrm{C}$ for $7 \mathrm{~min}$. c) Absorption spectrum of a HgTe nanocrystal film capped with EDT before and after annealing at $120^{\circ} \mathrm{C}$ for $7 \mathrm{~min}$. d) Mercury $4 f$ core levels of films prepared by EDT ligand exchange and by ink, just after their fabrication $(t=0)$, and after 72 hours spent in air $(t=72 \mathrm{~h})$. e) Tellurium $3 d$ core levels of films prepared by EDT ligand exchange and by ink, just after their fabrication $(t=0)$, and after 72 hours spent in air $(t=72 \mathrm{~h})$. On EDT capped HgTe film, after 72 hours in air, we see a second contribution, in red on the signal, highlighted by the black dotted lines.

This thick absorbing layer was then integrated into a photodiode, see Figure 3a-c. The diode has the following structure: glass/FTO/HgTe $4 \mathrm{k} / \mathrm{HgTe} 6 \mathrm{k} / \mathrm{Au}$. In the extended SWIR, the absorption of conventional transparent conductive oxide (TCO) starts to be non-negligible. To avoid an excessive absorption, a thin ( $\approx 80 \mathrm{~nm}$ ) fluorinated tin oxide (FTO) is used, its transmission at the band edge of the nanocrystals being above $60 \%$, see figure S6. A thick layer $(\approx 600 \mathrm{~nm})$ of HgTe $4 \mathrm{k}$ is used as an absorbing material, see Figure $3 \mathrm{~b}$ and $\mathrm{c}$. The diode rectifying behavior is introduced by a unipolar barrier made of HgTe nanocrystals with a wider band gap as proposed by Jagtap et al. ${ }^{[29]}$ The role of the unipolar barrier is to let photocurrent flow while preventing the dark current injection for the other carrier ${ }^{[39-41]}$. 
To reach this goal, the barrier's and absorbing material's electronic levels needed to be aligned for the carriers to flow (in this case, holes), while the electronic levels need to be offset for the carriers to be filtered (i.e. electon). Such barrier can be obtained with smaller HgTe nanocrystals exhibiting a band edge energy of $\approx 720 \mathrm{meV}$ ( $6000 \mathrm{~cm}^{-1}$ - later called HgTe $6 \mathrm{k}$ ). Ultraviolet photoemission (Figure S1d) reveals a near resonance between their valence band and that of the absorbing layer made of $\mathrm{HgTe} 4 \mathrm{k}$, while the conduction band is offset by more than $200 \mathrm{meV}$. It is also worth noticing that the HgTe 6k CQDs display only a p-type conduction (Figure S1) which further favors electron filtering. A top gold contact is used as a hole extractor.

The I-V curve reveals a rectifying behavior even at room temperature (see Figure $3 \mathrm{~d}$ ), with a weak dark current $\left(<10^{-6} \mathrm{~A} . \mathrm{cm}^{-2}\right)$ around $0 \mathrm{~V}$. Under black body illumination $(65 \mu \mathrm{W}$, corresponding to integrated power from $1.9 \mu \mathrm{m}$ to the HgTe $4 \mathrm{k}$ cut-off), the responsivity of the device has been determined to be $20 \mathrm{~mA} . \mathrm{W}^{-1}$, see Figure $3 e$ and S9. This is almost two orders of magnitude higher than previously reported devices at the same wavelength based on HgTe nanocrystals, and only diodes with optical resonators ${ }^{[19]}$ have achieved higher responsivities. This is the result of (i) a higher absorption thanks to a thicker HgTe film. $A \mathrm{~L}=600 \mathrm{~nm}$ thick film is expected to absorb $1-10^{-\alpha L} \approx$ $33 \%$ of light for each time it passes through the film. Experimentally, we determine that a film of $600 \pm 20 \mathrm{~nm}$ has an optical density of 0.23 at $4000 \mathrm{~cm}^{-1} \mathrm{~m}(2.5 \mu \mathrm{m})$, which corresponds to a light absorption of $40 \%$, see Figure S2. (ii) Better photocharge extraction due to the removal of the $\mathrm{TiO}_{2}$ layer. For the same diode structure, we observe a drop by four orders of magnitude of the responsivity in the presence of the $\mathrm{TiO}_{2}$ electron transport layer, see Figure S7. It is also worth noting that the frequency response of the device is flat in the $10 \mathrm{~Hz}$ to $1 \mathrm{kHz}$ range, suggesting a fast response time (see Figure 3e). ${ }^{[45]}$ The time response has actually been determined to be $\tau \approx 370 \mathrm{~ns}$ under nanosecond pulse excitation (Figure $2 \mathrm{~g}$ ), this corresponds to a cut-off frequency of $f_{c}=\frac{1}{2 \pi \tau}=$ $430 \mathrm{kHz}$.

From this long lifetime, we can estimate the carrier diffusion length $L_{D}^{[46]}$ to be given by $L_{D}=\sqrt{\frac{\mu k_{b} T \tau}{e}}$ with $\mu$ the mobility, $k_{b}$ the Boltzman constant, $T$ the temperature and e the proton charge. Using the value of $10^{-4} \mathrm{~cm}^{2} \mathrm{~V}^{-1} \mathrm{~s}^{-1}$, as measured for EDT capped HgTe nanocrystals, we obtain a $10 \mathrm{~nm}$ diffusion length, barely larger than the nanocrystal size. Fabricating thick film is not useful in this condition since photocarriers will not be collected. On the other hand, the ink, with its 300 times higher mobility, leads to diffusion length of $\approx 170 \mathrm{~nm}$, which can lead to an efficient charge collection over the $500 \mathrm{~nm}$ thick diode film.

Again, for nanocrystal based devices, the noise is found to be $1 / f$ limited, ${ }^{[47-49]}$ (see the inset of Figure $3 \mathrm{f}$ and Figure $\mathrm{S10}$ ). The detectivity is calculated as follows: $D^{*}=\frac{R \sqrt{A}}{i_{n}}$ with $R$ the responsivity in A.W $W^{-1}, A$ the optical area of the device and $i_{n}$ the current spectral density in $\mathrm{A} . \mathrm{Hz}^{-1 / 2}$. The measured detectivity reaches $3 \times 10^{9}$ Jones at room temperature, under $0 \mathrm{~V}$ and for detection at $1 \mathrm{kHz}$ (see Figure 2f). 

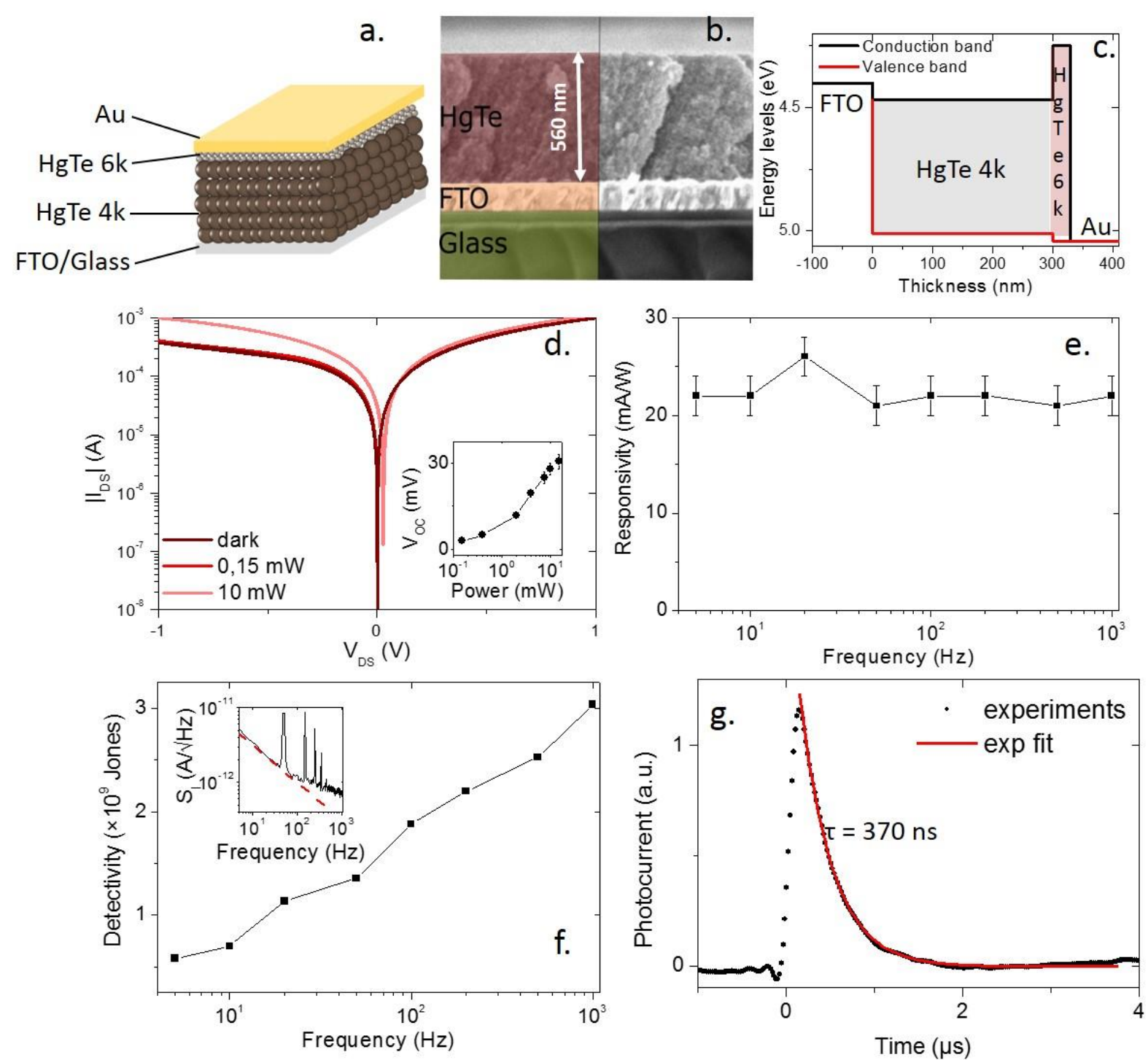

Figure 3: a) Diagram of the diode structure. b) SEM cross-section of the diode. c) Band alignment of the different components of the diode. d) IV curves in the dark and under illumination by a $1.55 \mu \mathrm{m}$ laser at different powers, insert: evolution of open circuit voltage ( $\left.V_{O C}\right)$ as a function of increasing power. e) Responsivity under black body illumination at $980{ }^{\circ} \mathrm{C}$, corresponding to $65 \mu \mathrm{W}$ incident power, at different frequencies. A Ge filter is used to remove photons with a wavelength below 1.9 $\mu \mathrm{m}$, as well as to ensure that the responsivity only comes from $\mathrm{HgTe} 4 \mathrm{k}$ excitation. f) Detectivity as a function of frequency, insert: noise signal as a function of detection frequency (black line) and $f^{-1 / 2}$ fit (red dash line). g) Time response of the diode measured with $355 \mathrm{~nm}$ pulsed laser (2 ns pulse). The time response value is obtained through single exponential fitting.

The operation of the diode and the unipolar barrier can be better appreciated thanks to photocurrent spectra (see Figure 4a). Since the unipolar barrier is made from an active material (HgTe 6k), photocurrent is likely to occur from different parts of the diode under broad band excitation. In the reverse bias regime, the HgTe $6 \mathrm{k}$ barrier prevents electron injection from the gold side (i.e., dark current) but does not prevent electronic current coming from HgTe $4 \mathrm{k}$ excitation (i.e., photocurrent) nor hole current (see the left inset of Figure $4 \mathrm{~b}$ ). This tends to maximize the photocurrent resulted from the HgTe $4 \mathrm{k}$ layer. Under low forward bias, a significant part of the 
electronic photocurrent coming from the HgTe $4 \mathrm{k}$ layer gets blocked by the barrier. A decrease of the relative weight of $\mathrm{HgTe} 4 \mathrm{k}$ in the photocurrent was observed (see the middle inset of Figure $4 \mathrm{~b}$ ). When a large bias is applied, the Stark effect increases and thus the transparency of the barrier and the photocurrent from the HgTe $4 \mathrm{k}$ layer can again flow in the system (see the right inset of Figure 4b). In this sense, the unipolar barrier plays exactly its expected role as long as the device is operated under low voltage. This happens when the energy drops along the barrier e.F.L (where $e$ is the elementary charge, $F$ is the applied electric field and $L$ is the length of the barrier, around $30 \mathrm{~nm}$ ) is far below the band offset between $\mathrm{HgTe} 4 \mathrm{k}$ and HgTe $6 \mathrm{k}(\approx 200 \mathrm{meV})$
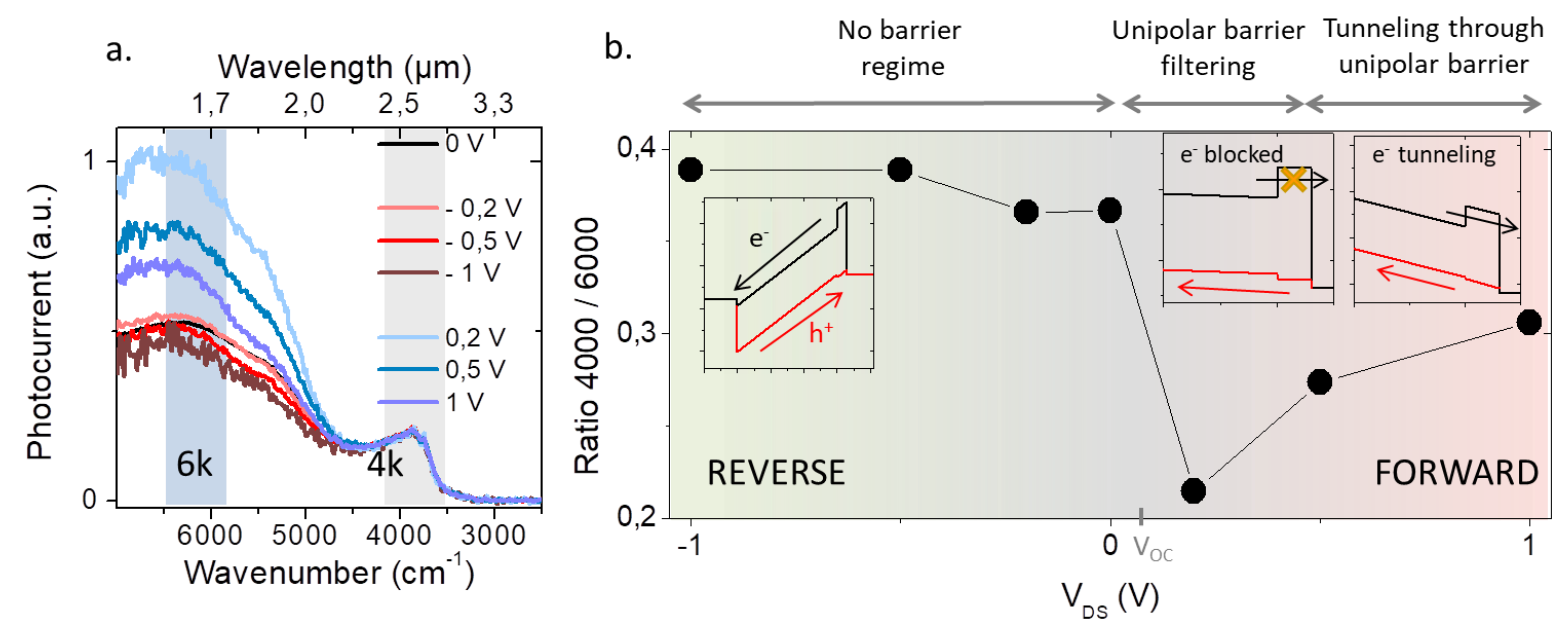

Figure 4: a) Photocurrent spectra of the diode under different biases. b) Measured ratio of photocurrent at $4000 \mathrm{~cm}^{-1}$ by photocurrent at $6000 \mathrm{~cm}^{-1}$. as a function of applied bias, inserts: band diagrams at different biases. The black line is the conduction band, the red line is the valence band. The black and red arrows correspond to the electron and hole trajectories respectively.

The diode capability for low flux detection has been tested by detecting a black body far away from the source $(\approx 4 \mathrm{~m}$, see Figure $5 \mathrm{a}$ and $\mathrm{c})$. It is worth mentioning that extended SWIR diodes are not optimized for thermal imaging since only the photons in the abrupt rise of the black body spectrum between the Ge filter cutoff ( $1.9 \mu \mathrm{m}$ to suppress visible photon) and the diode cut off wavelength $(2.5 \mu \mathrm{m})$ get absorbed. At this distance, warm black bodies $\left(>700{ }^{\circ} \mathrm{C}\right)$ can be clearly discriminated from the dark current (see Figure 5b). Another important characteristic, often overlooked for nanocrystal-based devices, is the angular response determination. The latter well follows the expected cosine dependency (see Figure $5 \mathrm{~d}$ ). As it may have been anticipated, though not confirmed, no angular selection rules are introduced by the HgTe nanocrystals or by any waveguide effect within the diode stacking. 

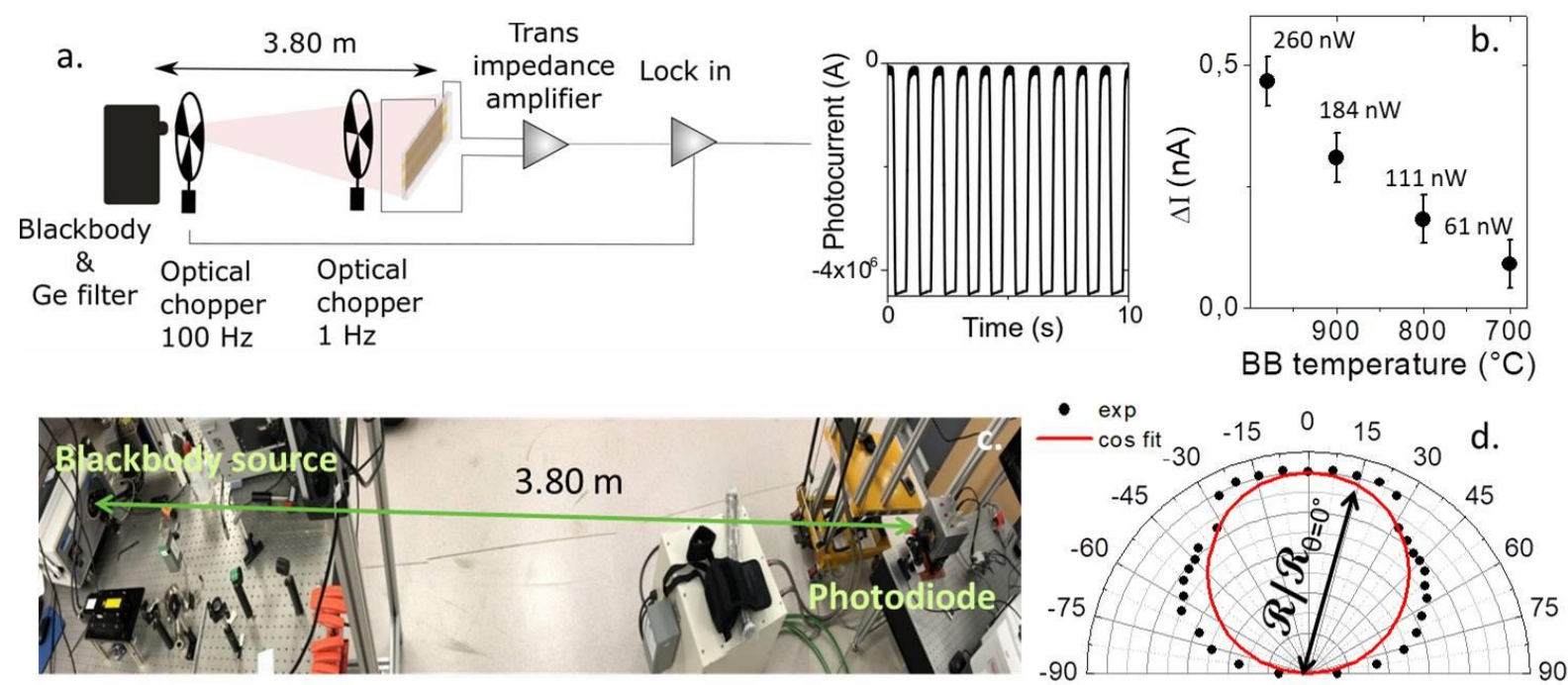

Figure 5: a) Set up schematic for long distance detection. The measured signal is the $100 \mathrm{~Hz}$ current contribution modulated at $1 \mathrm{~Hz}$. b) Signal current measured at different black body (BB) temperatures. c) Photograph of the experimental set up. d) Angular detection with a $1.55 \mu \mathrm{m}, 4 \mathrm{~mW}$ laser. The black dots correspond to the experimental ratio between the responsivity at a given angle and at $0^{\circ}$ (i.e. normal incidence), the red circle corresponds to a cosine evolution.

The last part of the paper is dedicated to the integration of this strongly absorbing film of HgTe within a multipixel geometry. $\mathrm{HgTe}$, as all $\mathrm{Hg}$ containing materials, is relatively soft and the nanocrystals' growth temperature is relatively low $\left(80^{\circ} \mathrm{C}\right)$ making them easy to sinter when exposed to high temperatures. This leads to a redshift of the optical feature and a dramatic increase of the dark current. ${ }^{[28]}$ The latter process can be partly prevented by using $\mathrm{HgTe} / \mathrm{CdTe}$ core-shell systems. ${ }^{[50]}$ Regardless, it remains that device geometries and fabrication steps need to account for the material's sensibility. Here, we demonstrate the feasibility of a $10 \times 10$ pixel matrix and its use for infrared laser beam shape determination. To limit the exposition of the HgTe nanocrystals to any annealing step, the electrodes are built $a$ priori and the nanocrystals are deposited during the last step of fabrication. The matrix fabrication is described in Figure 6, S11 and S12. Typically, each pixel has a $27 \mu \mathrm{m}$ size, with a $33 \mu \mathrm{m}$ size, corresponding to a filling factor of $67 \%$ over the whole matrix. One should note that this pixel size is similar to what is currently used for InGaAs technology. Each pixel is made up of two metallic contacts separated by a $\mathrm{SiO}_{2}$ insulating barrier. 

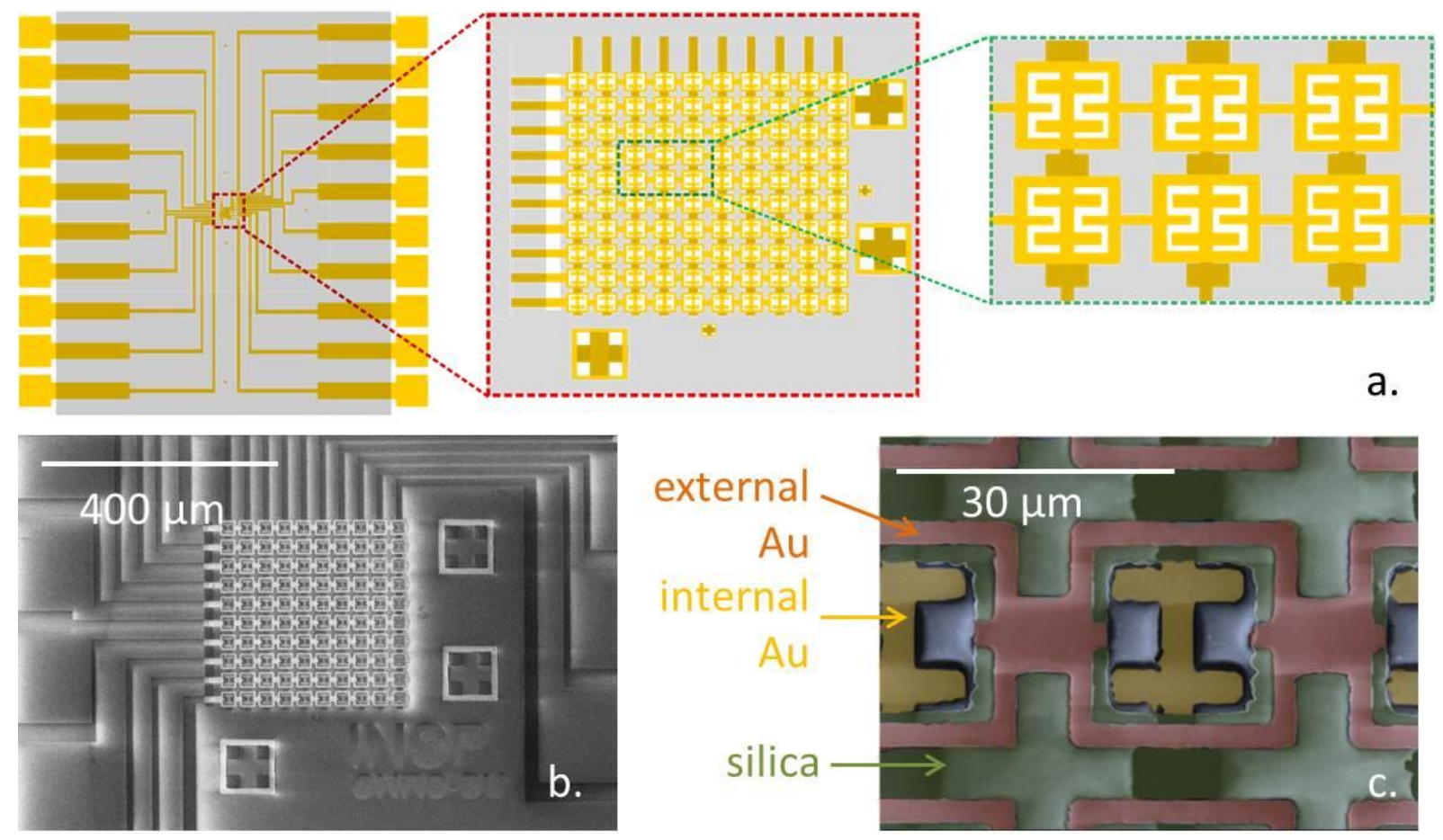

Figure 6 a) Schematic of the matrix at different zoom levels. b) SEM image of the matrix (magnified 200x). c) SEM image of the matrix with faux colors corresponding to different layers: yellow is the internal gold contact (columns), green is the insulating silica and red is the external gold contact (lines) (magnified 3000x). Blue relates to the glass substrate.

The matrix' homogeneity can be seen from the dark current histogram, see Figure 7a. Out of 100 pixels, only 3 were shorted (even before the ink deposition). The full width at half maximum (FWMH) of the distribution is $45 \%$ of the average value. This may seem important, however, the fluctuations are mostly the result of the electrodes dispersion quality rather than film quality since we can spatially correlate the less resistive pixels to be in the vicinity of the shorted electrodes. 

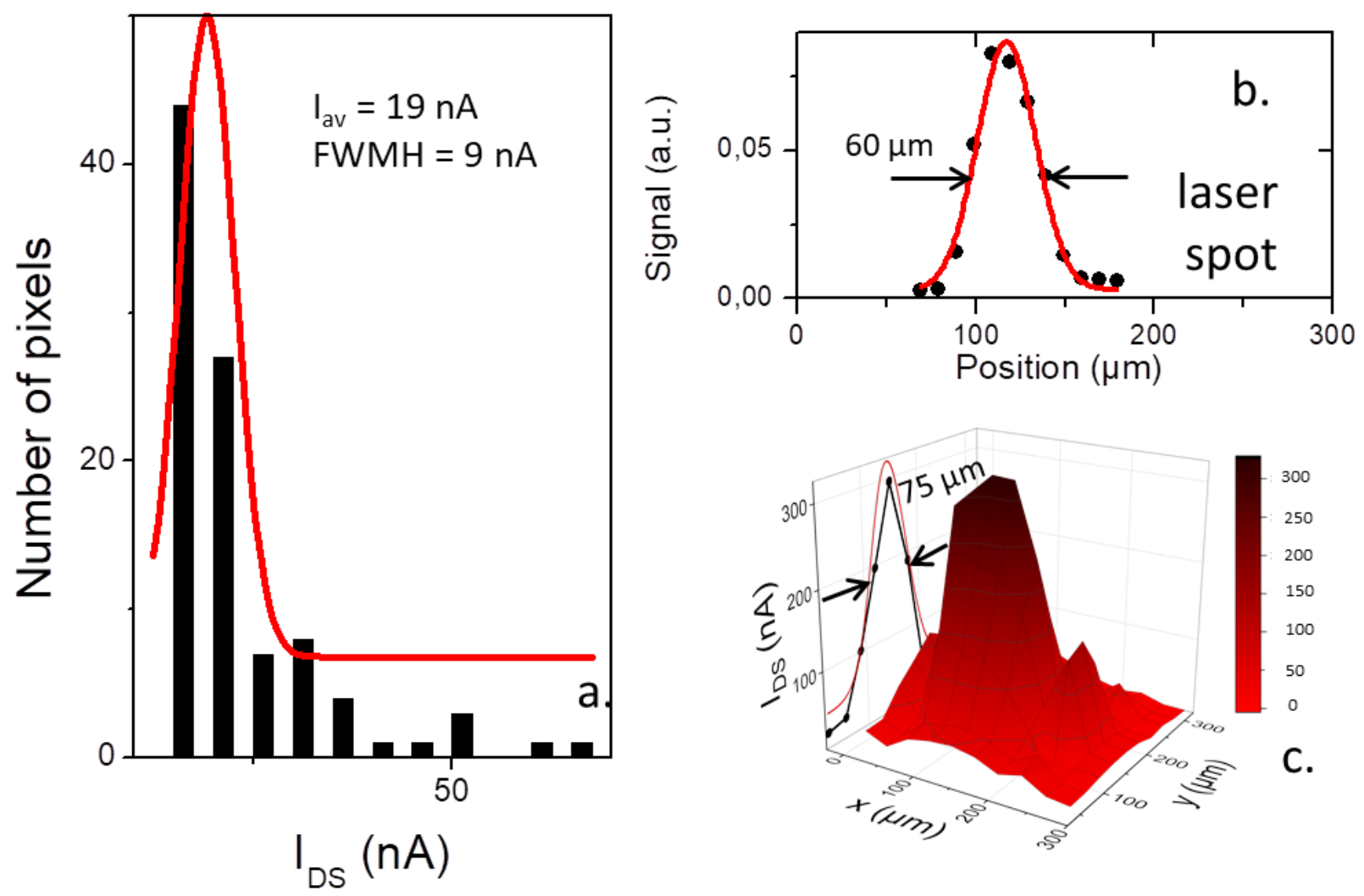

Figure 7: Matrix response. a) Dark current under $0.1 \mathrm{~V}$ for all the pixels on the matrix except the three shorted. b) Determination of $1.55 \mu \mathrm{m}$ focused laser spot size using a razor blade. The experimental points are the black dots, the Gaussian fit is the red line. c) Intensity of the 97 working pixels with laser spot focused on the matrix. The Gaussian shape has a full width at half maximum (FWMH) of 75 $\mu m$.

We have then tested the capability of this focal plane array (FPA) for infrared beam shape determination. We have used a $1.55 \mu \mathrm{m}$ laser diode source, with a beam linewidth determined to be around $60 \mu \mathrm{m}$, see Figure 7b. Once the laser is shone on the FPA, we recovered a similar linewidth 75 $\mu \mathrm{m}$, see Figure 7c. Hence, the FPA is sensitive enough to detect higher order modes present in the laser beam, see Figure S13.

\section{CONCLUSION}

We have explored the possibility to use HgTe nanocrystal-based ink to design extended short wave infrared sensors with enhanced absorption. Compared to solid state ligand exchange film, the ink preparation preserves the optical spectrum and leads to higher electrical stability for in air operation. The material has been integrated into a diode, where the rectifying behavior is the result of a unipolar barrier. With this geometry, we have improved the responsivity of the film by two orders of magnitude and the detectivity by one order of magnitude compared to previous devices at the same wavelength and based on the same material. The detectivity now reaches $3 \times 10^{9}$ Jones at room temperature, while the time response can be as short as $370 \mathrm{~ns}$. The material has also been tested as an active material of a focal plane array (FPA), with a pixel pitch down to $30 \mu \mathrm{m}$, with the fabrication designed to account for HgTe softness. We demonstrate the use of this FPA for infrared laser beam shape determination. 


\section{Supporting information}

Additional information about nanocrystal synthesis, material characterization, diode fabrication and characterization as well as focal plane array fabrication and characterization are given in supporting information.

Supporting Information is available from the Wiley Online Library.

\section{ACKNOWLEDEGMENTS}

EL thanks the support ERC starting grant blackQD (grant $n^{\circ} 756225$ ). We acknowledge the use of clean-room facilities from the "Centrale de Proximité Paris-Centre". This work has been supported by the Region Ile-de-France in the framework of DIM Nano-K (grant dopQD). This work was supported by French state funds managed by the ANR within the Investissements d'Avenir program under reference ANR-11-IDEX-0004-02, and more specifically within the framework of the Cluster of Excellence MATISSE and also by the grant IPER-nano2. NG and JR thank Nexdot for post doc funding.

\section{REFERENCES}

[1] Talapin, Dmitri V., Lee, Jong Soo, Kovalenko, Maksym V., Shevchenko, Elena V., Chem Rev 2010, 110, 389.

[2] G. Konstantatos, E. H. Sargent, Colloidal Quantum Dot Optoelectronics and Photovoltaics, Cambridge University Press, 2013.

[3] V. I. Klimov, Nanocrystal Quantum Dots, 2nd Edition, CRC Press, 2010.

[4] K. Bourzac, Nature 2013, 493, 283.

[5] X. Brokmann, E. Giacobino, M. Dahan, J. P. Hermier, Appl. Phys. Lett. 2004, 85, 712.

[6] B. Dubertret, P. Skourides, D. J. Norris, V. Noireaux, A. H. Brivanlou, A. Libchaber, Science 2002, 298, 1759.

[7] E. Lhuillier, M. Scarafagio, P. Hease, B. Nadal, H. Aubin, X. Z. Xu, N. Lequeux, G. Patriarche, S. Ithurria, B. Dubertret, Nano Lett. 2016, 16, 1282.

[8] N. Goubet, A. Jagtap, C. Livache, B. Martinez, H. Portalès, X. Z. Xu, R. P. S. M. Lobo, B. Dubertret, E. Lhuillier, J. Am. Chem. Soc. 2018, 140, 5033.

[9] G. Konstantatos, I. Howard, A. Fischer, S. Hoogland, J. Clifford, E. Klem, L. Levina, E. H. Sargent, Nature 2006, 442, 180.

[10] D. C. Oertel, M. G. Bawendi, A. C. Arango, V. Bulović, Appl. Phys. Lett. 2005, 87, 213505.

[11] H. Chen, H. Liu, Z. Zhang, K. Hu, X. Fang, Adv. Mater. 2016, 28, 403.

[12] Y. Bi, S. Pradhan, S. Gupta, M. Z. Akgul, A. Stavrinadis, G. Konstantatos, Adv. Mater. 2018, 30, 1704928.

[13] S. Yakunin, D. N. Dirin, L. Protesescu, M. Sytnyk, S. Tollabimazraehno, M. Humer, F. Hackl, T. Fromherz, M. I. Bodnarchuk, M. V. Kovalenko, W. Heiss, ACS Nano 2014, 8, 12883.

[14] J. M. Pietryga, R. D. Schaller, D. Werder, M. H. Stewart, V. I. Klimov, J. A. Hollingsworth, J. Am. Chem. Soc. 2004, 126, 11752.

[15] N. Huo, S. Gupta, G. Konstantatos, Adv. Mater. 2017, 29, 1606576.

[16] S. E. Keuleyan, P. Guyot-Sionnest, C. Delerue, G. Allan, ACS Nano 2014, 8, 8676. 
[17] M. V. Kovalenko, E. Kaufmann, D. Pachinger, J. Roither, M. Huber, J. Stangl, G. Hesser, F. Schäffler, W. Heiss, J. Am. Chem. Soc. 2006, 128, 3516.

[18] P. Guyot-Sionnest, J. A. Roberts, Appl. Phys. Lett. 2015, 107, 253104.

[19] X. Tang, M. M. Ackerman, G. Shen, P. Guyot-Sionnest, Small 2019, 15, 1804920.

[20] M. Chen, H. Lu, N. M. Abdelazim, Y. Zhu, Z. Wang, W. Ren, S. V. Kershaw, A. L. Rogach, N. Zhao, ACS Nano 2017, 11, 5614.

[21] M. Chen, L. Shao, S. V. Kershaw, H. Yu, J. Wang, A. L. Rogach, N. Zhao, ACS Nano 2014, 8, 8208.

[22] M. Chen, H. Yu, S. V. Kershaw, H. Xu, S. Gupta, F. Hetsch, A. L. Rogach, N. Zhao, Adv. Funct. Mater. 2014, 24, 53.

[23] X. Tang, X. Tang, K. W. C. Lai, ACS Photonics 2016, 3, 2396.

[24] M. E. Cryer, J. E. Halpert, ACS Photonics 2018, 5, 3009.

[25] M. M. Ackerman, X. Tang, P. Guyot-Sionnest, ACS Nano 2018, 12, 7264.

[26] X. Tang, M. M. Ackerman, P. Guyot-Sionnest, ACS Nano 2018, 12, 7362.

[27] X. Tang, G. F. Wu, K. W. C. Lai, J Mater Chem C 2017, 5, 362.

[28] A. Jagtap, N. Goubet, C. Livache, A. Chu, B. Martinez, C. Greboval, J. Qu, E. Dandeu, L.

Becerra, N. Witkowski, S. Ithurria, F. Mathevet, M. G. Silly, B. Dubertret, E. Lhuillier, J. Phys. Chem. C 2018, 122, 14979.

[29] A. Jagtap, B. Martinez, N. Goubet, A. Chu, C. Livache, C. Gréboval, J. Ramade, D. Amelot, P. Trousset, A. Triboulin, S. Ithurria, M. G. Silly, B. Dubertret, E. Lhuillier, ACS Photonics 2018, 5, 4569.

[30] E. Lhuillier, S. Keuleyan, P. Guyot-Sionnest, Nanotechnology 2012, 23, 175705.

[31] A. Fischer, L. Rollny, J. Pan, G. H. Carey, S. M. Thon, S. Hoogland, O. Voznyy, D. Zhitomirsky, J. Y. Kim, O. M. Bakr, E. H. Sargent, Adv. Mater. 2013, 25, 5742.

[32] D. M. Balazs, N. Rizkia, H.-H. Fang, D. N. Dirin, J. Momand, B. J. Kooi, M. V. Kovalenko, M. A. Loi, ACS Appl. Mater. Interfaces 2018, 10, 5626.

[33] Z. Ning, H. Dong, Q. Zhang, O. Voznyy, E. H. Sargent, ACS Nano 2014, 8, 10321.

[34] A. Nag, M. V. Kovalenko, J.-S. Lee, W. Liu, B. Spokoyny, D. V. Talapin, J. Am. Chem. Soc. 2011, $133,10612$.

[35] S. Keuleyan, E. Lhuillier, P. Guyot-Sionnest, J. Am. Chem. Soc. 2011, 133, 16422.

[36] B. Yoon, J. Jeong, K. S. Jeong, J. Phys. Chem. C 2016, 120, 22062.

[37] S. Ghosh, L. Manna, Chem. Rev. 2018, 118, 7804.

[38] J. Tang, K. W. Kemp, S. Hoogland, K. S. Jeong, H. Liu, L. Levina, M. Furukawa, X. Wang, R. Debnath, D. Cha, K. W. Chou, A. Fischer, A. Amassian, J. B. Asbury, E. H. Sargent, Nat. Mater. 2011, 10,765 .

[39] E. Lhuillier, S. Keuleyan, P. Zolotavin, P. Guyot-Sionnest, Adv. Mater. 2013, 25, 137.

[40] E. Lhuillier, S. Ithurria, A. Descamps-Mandine, T. Douillard, R. Castaing, X. Z. Xu, P.-L. Taberna, P. Simon, H. Aubin, B. Dubertret, J. Phys. Chem. C 2015, 119, 21795.

[41] M. K. Bahl, R. L. Watson, K. J. Irgolic, J. Chem. Phys. 1977, 66, 5526.

[42] P. Martyniuk, M. Kopytko, A. Rogalski, Opto-Electron. Rev. 2014, 22, 127.

[43] G. R. Savich, J. R. Pedrazzani, D. E. Sidor, S. Maimon, G. W. Wicks, Appl. Phys. Lett. 2011, 99, 121112.

[44] G. R. Savich, J. R. Pedrazzani, D. E. Sidor, G. W. Wicks, Infrared Phys. Technol. 2013, 59, 152.

[45] C. Livache, N. Goubet, B. Martinez, A. Jagtap, J. Qu, S. Ithurria, M. G. Silly, B. Dubertret, E. Lhuillier, ACS Appl. Mater. Interfaces 2018, 10, 11880. 
[46] W. J. Mir, C. Livache, N. Goubet, B. Martinez, A. Jagtap, A. Chu, N. Coutard, H. Cruguel, T. Barisien, S. Ithurria, A. Nag, B. Dubertret, A. Ouerghi, M. G. Silly, E. Lhuillier, Appl. Phys. Lett. 2018, 112, 113503.

[47] A. De lacovo, C. Venettacci, L. Colace, L. Scopa, S. Foglia, Appl. Phys. Lett. 2017, 111, 211104.

[48] Y. Lai, H. Li, D. K. Kim, B. T. Diroll, C. B. Murray, C. R. Kagan, ACS Nano 2014, 8, 9664.

[49] H. Liu, E. Lhuillier, P. Guyot-Sionnest, J. Appl. Phys. 2014, 115, 154309.

[50] G. Shen, P. Guyot-Sionnest, Chem. Mater. 2019, 31, 286. 